\title{
A Rare Case of Idiopathic Ovarian Vein Thrombosis
}

Shauna Kern, DO and Jill Hanrahan, MD

\section{Introduction}

Ovarian vein thrombosis most commonly occurs in the postpartum period. The incidence ranges from 1 in 600 to 1 in 2000 deliveries. Other associations include malignancy, surgery, pelvic inflammatory disease, sepsis, and hypercoagulable states. This condition can have potentially dangerous complications including pulmonary embolism, sepsis, extension into the inferior vena cava or renal veins, and ovarian infarction. $^{2}$ For these reasons, it is important to include ovarian vein thrombosis in the differential diagnosis for abdominal pain.

\section{Case History}

A 34-year-old Caucasian female with history of impaired glucose tolerance and depression presented with a one-week history of right-sided low back pain. She also reported nausea and lower abdominal cramping.

She reported no recent surgeries, no use of birth control, and her family history was unknown due to being adopted.

Pertinent exam findings included tenderness to palpation in the right lower back and right lower quadrant. A pelvic exam was normal. Initial basic laboratories and pelvic swabs were unremarkable. However, CT of the abdomen/pelvis with contrast revealed a right ovarian vein thrombosis.

The patient was treated with warfarin for 6 months. Upon completion, further testing showed no evidence of a hypercoaguable state.

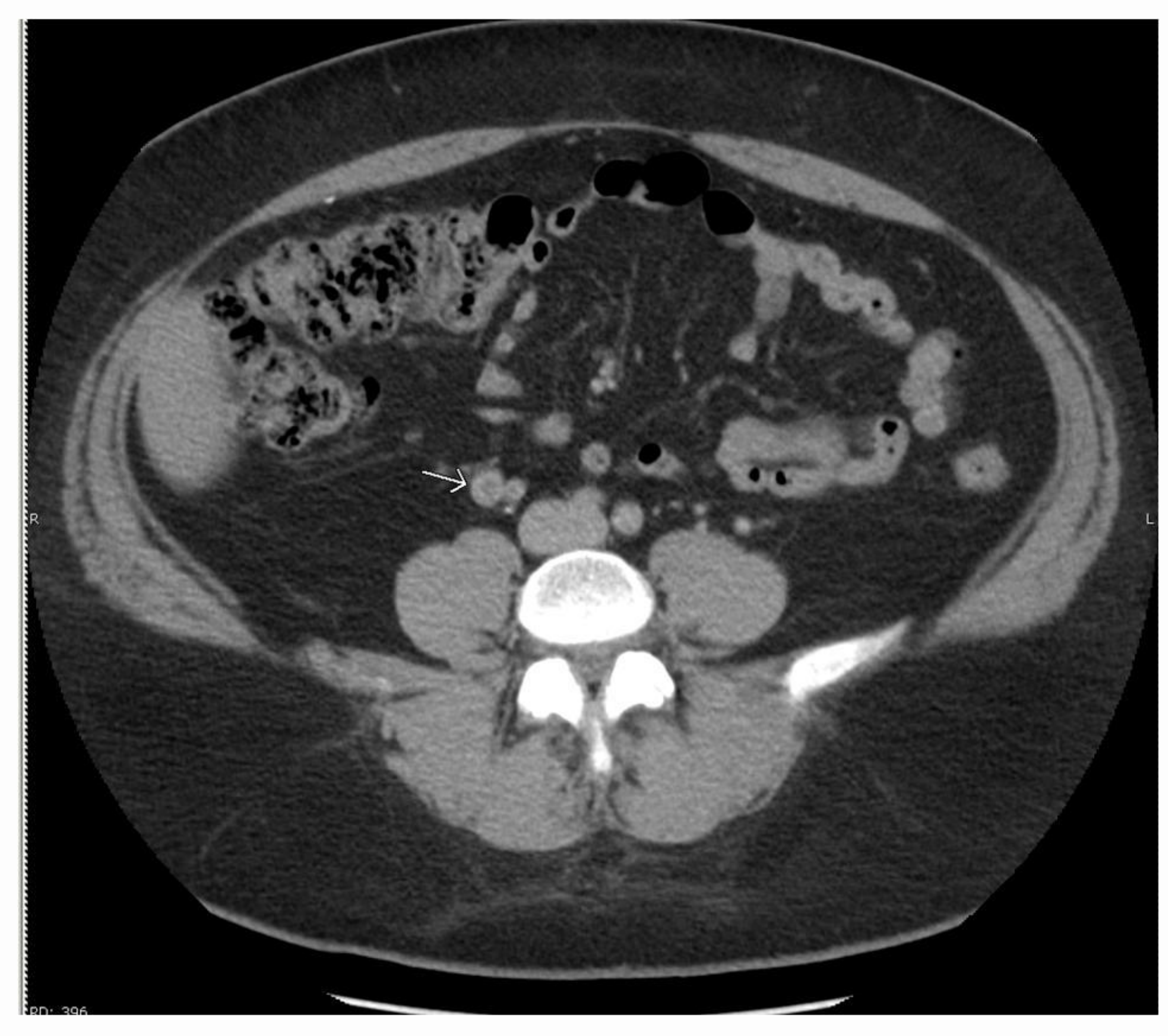

CT scan depicts an enlarged right ovarian vein with a filling defect indicative of ovarian vein thrombosis.

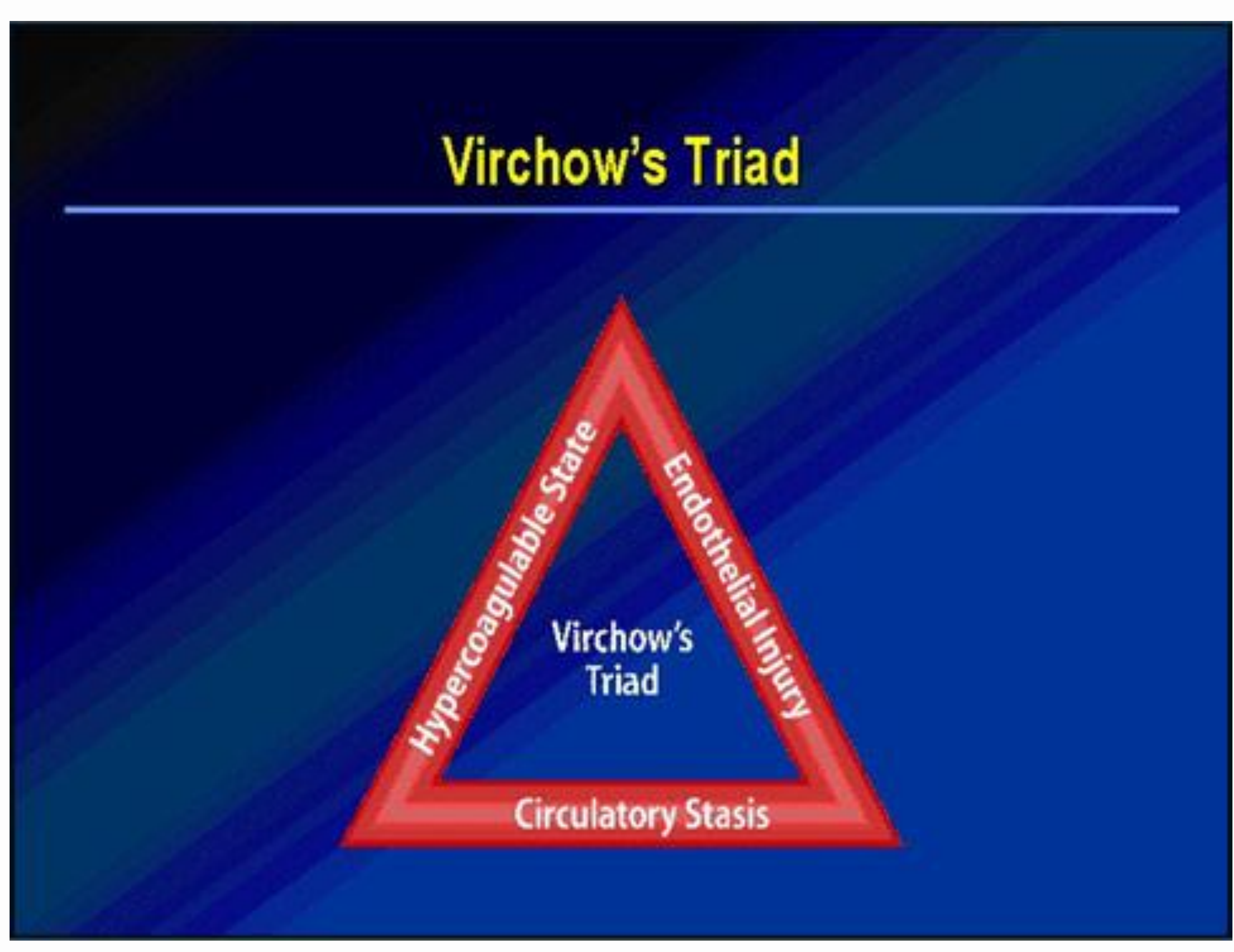

\section{Discussion}

This case involved none of the usual associations with ovarian vein thrombosis. In searching the literature, only three cases of idiopathic ovarian vein thrombosis have been noted, including one each in Turkey, the United States, and New Zealand. ${ }^{1}$

The most common presenting signs are fever and right iliac fossa pain. Lower abdominal pain and/or a palpable abdominal mass may be present on exam. ${ }^{2}$ CT and MRI may help to confirm the diagnosis. Ultrasound does not always clearly demonstrate thrombosis in the ovarian vein. ${ }^{1}$

Six months of anticoagulation is the standard treatment in all reported cases. Repeat imaging is useful to assure resolution of the clot. ${ }^{2}$

\section{$\underline{\text { References }}$}

1. Stafford, M Idiopathic Ovarian Vein Thrombosis: A Rare Cause of Pelvic Pain - Case Report and Review of Literature. Austrailian and New Zealand Journal of Obstetrics and Gynecology. Volume 50(3): 299-301. June 2010.

2. Yildirim, E. Isolated Idiopathic Vein Thrombosis: A Rare Case. International Urogynecology Journal. Volume 16; 308310. 2005. 